\title{
Phylogeny and polymorphism in the long control regions E6, E7, and L1 of HPV Type 56 in women from southwest China
}

\author{
YALING JING ${ }^{1,2^{*}}$, TAO WANG ${ }^{1,2^{*}}$, ZUYI CHEN $^{1,2}$, XIANPING DING ${ }^{1,2}$, \\ JIANJU XU ${ }^{1,2}$, XUEMEI MU ${ }^{1,2}$, MAN CAO ${ }^{1,2}$ and HONGHAN CHEN ${ }^{1,2}$ \\ ${ }^{1}$ Key Laboratory of Bio-Resources and Eco-Environment, Ministry of Education, Institute of Medical Genetics, \\ College of Life Science, Sichuan University, Chengdu, Sichuan 610064; ${ }^{2}$ Bio-Resource Research and \\ Utilization Joint Key Laboratory of Sichuan and Chongqing, Chengdu, Chongqing 408400, P.R. China
}

Received September 3, 2016; Accepted July 27, 2017

DOI: $10.3892 / \mathrm{mmr} .2018 .8743$

\begin{abstract}
Globally, human papillomavirus (HPV)-56 accounts for a small proportion of all high-risk HPV types; however, HPV-56 is detected at a higher rate in Asia, particularly in southwest China. The present study analyzed polymorphisms, intratypic variants, and genetic variability in the long control regions (LCR), E6, E7, and L1 of HPV-56 $(n=75)$. The LCRs, E6, E7 and L1 were sequenced using a polymerase chain reaction and the sequences were submitted to GenBank. Maximum-likelihood trees were constructed using Kimura's two-parameter model, followed by secondary structure analysis and protein damaging prediction. Additionally, in order to assess the effect of variations in the LCR on putative binding sites for cellular proteins, MATCH server was used. Finally, the selection pressures of the E6-E7 and L1 genes were estimated. A total of 18 point substitutions, a 42-bp deletion and a 19-bp deletion of LCR were identified. Some of those mutations are embedded in the putative binding sites for transcription factors. 18 single nucleotide changes occurred in the E6-E7 sequence, 11/18 were non-synonymous substitutions and $7 / 18$ were synonymous mutations. A total 24 single nucleotide changes were identified in the L1 sequence, 6/24 being non-synonymous mutations and $18 / 24$ synonymous mutations. Selective pressure analysis predicted that the majority of mutations of HPV-56 E6, E7 and L1 were of positive selection. The phylogenetic tree demonstrated that the isolates distributed in two lineages. Data on the prevalence and genetic variation of
\end{abstract}

Correspondence to: Professor Xianping Ding, Key Laboratory of Bio-Resources and Eco-Environment, Ministry of Education, Institute of Medical Genetics, College of Life Science, Sichuan University, 24 South Part First Ring Road, Wu Hou, Chengdu, Sichuan 610064, P.R. China

E-mail: brainding@scu.edu.cn

${ }^{*}$ Contributed equally

Key words: human papillomavirus-56, gene polymorphism, phylogenetic trees, selection pressures
HPV-56 types in southwest China may aid future studies on viral molecular mechanisms and contribute to future investigations of diagnostic probes and therapeutic vaccines.

\section{Introduction}

Cervical cancer, the fourth most common cancer in women worldwide, with relatively high numbers occurring in developing countries, including China, has the major etiological factor which is persistent infection of oncogenic Human papillomavirus (HPV) (1,2).

The HPV genome is a small double-stranded circular DNA molecule of 8,000 base pairs (bp) which contains three regions: i) 4,000 bp region encoding 6 early proteins participating in viral replication and cell transformation, including 3 regulatory proteins (E1, E2 and E4) and 3 oncoproteins (E5, E6 and E7); ii) 3,000 bp region encoding the structural proteins of the virus, including major capsid protein L1 and the minor capsid protein L2; and iii) 1,000 bp long control region (LCR) that contains the origin of viral DNA replication and transcriptional regulatory elements (3).

Any two HPV strains may be classified into types, subtypes or variants according to the difference rate between their L1 sequences that is the difference rate $>10 \%$, between 2 and $10 \%$ or $<2 \%$ (4). Currently, $>200$ HPV types have been fully characterized (http://www.hpvcenter.se/html/refclones. html), and the International Agency for Research on Cancer defined 12 HPV types as oncogenic or potentially carcinogenic groups (5). Except for HPV51 (belongs to a-5) and HPV-56 (belongs to a-6), others of these carcinogenic types are phylogenetically clustered with a-7 (HPV-18, HPV-39, HPV-45, HPV-59) or a-9 (HPV-16, HPV-31, HPV-33, HPV-35, HPV-52, HPV-58) (6).

HPV-56 is an oncogenic HPV type, which was initially described in the United States in 1989 (7). Globally, HPV-56 accounts for $<1 \%$ of cervical cancer cases (1) However, compared with the rest of the world, the prevalence of HPV-56 is remarkably high in Asia, particularly in China $(8,9)$. According to a recent study from China's largest KingMed Laboratory (Guangzhou, Guangdong, China), from January 2011 to June 2014, the detection rate of HPV-56 among the 51,345 samples was ranked fourth below the three most highly detected types, HPV-52, HPV-16, and HPV-58 (10). 
Additionally, another previous study on the prevalence and distribution of HPV in 37 cities in China revealed that HPV-56 infection rate is $6.09 \%$, ranking fifth from 2,580 high risk HPV-positive samples (11). These findings are similar to those observed in southwest China in the past two years (12).

Previous studies suggested that variants of the same HPV type are biologically distinct and may result in differing pathogenic risks (13-15). The viral LCR region as the primary recognition site of the transcription factor, variations in this region may affect virus replication rates and the transcriptional activity (16). When considering the E6 and E7 oncoproteins, one or more amino acid changes may potentially alter cellular immortalization, transformation and carcinogenesis $(17,18)$. Changes in the L1 amino acid sequence, which contains sequences important for conformation of viral neutralization-relevant epitopes, may affect viral antigenicity or influence the effectiveness of viral infection (19). Currently, the information available on sequence variation in HPV-16, HPV-18, HPV-52, and HPV-58 is relatively comprehensive (20-23). However, data on HPV-56 variants is limited and sequence variations of HPV-56 in the Asian population remain to be elucidated $(24,25)$. Therefore, the aim of the present study was to detect sequence variations within LCR, E6, E7, and L1 of HPV-56 in women from southwest China. The current study may improve the understanding of the viral persistence, transmission and oncogenic potential. Furthermore, identifying novel variants of HPV-56 may assist in the design of vaccines and development of diagnostic probes for specific populations.

\section{Materials and methods}

Ethics statement. The present study was approved by the Education and Research Committee and the Ethics Committee of Sichuan University (Chengdu, China). Prior to sample collection, written informed consent was obtained from the patients and study subject privacy was carefully protected.

Sample collection. From January 2014 to January 2016, 7,707 cervical swabs were collected from female patients (age range, 18-65 years) that received HPV infection screening at the following maternity hospitals in Sichuan, China: Sichuan Reproductive Health Research Center Affiliated Hospital, The Angel Women's and Children's Hospital, The Chengdu Western Hospital Maternity Unit, The Peoples' Hospital of Pengzhou, Jinjiang Maternity and Child Health Hospital, Chengdu Zongnan Gynecology Hospital and Chengdu Songziniao Sterility Hospital. Samples were placed in preservative buffer (Yaneng Bioscience Co., Ltd., Shenzhen, China) and stored at $-20^{\circ} \mathrm{C}$.

HPV DNA detection and typing. Following sample collection, the viral genomic DNA was extracted and genotyped using the human papillomavirus genotyping kit for 23 types (Yaneng Bioscience Co., Ltd.) according to the manufacturer's protocol. This kit is based on a reverse dot blot principle and is able to classify 23 HPV genotypes, including the 18 high-risk, the probable high-risk types $(16,18,31,33,35,39,45,51,52$, $53,56,58,59,66,68,73,83$, and MM4), and the 5 low-risk types $(6,11,42,43$, and 44$)$. A total of 101 of HPV-56 positive samples DNA were detected and subsequently stored at $-20^{\circ} \mathrm{C}$.
PCR amplification and variant identification. The LCR fragment was amplified using specific primers as previously described (24). The complete genes of E6, E7 and L1 primers were designed using Primer Primier version 5.0 (Premier Biosoft, Palo Alto CA, USA; http://www.premierbiosoft .com/) (26) based on the HPV-56 GenBank reference sequences X74483 (accession no. X74483; www.ncbi.nlm.nih.gov/ nuccore/X74483) and listed in Table I. The primers were synthesized by Sangon Biotech Co., Ltd., (Shanghai, China).

Each $25 \mu \mathrm{l}$ PCR reaction contained $3 \mu \mathrm{l}$ of extracted DNA (10-100 ng), $2.5 \mathrm{mM}$ 10X PCR buffer $\left(\mathrm{Mg}^{2+}\right)$ (Beijing TransGen Biotech Co., Ltd., Beijing, China), $2.5 \mathrm{mM}$ dNTPs (Beijing TransGen Biotech Co., Ltd.), 2U Taq DNA polymerase (Sangon Biotech Co., Ltd.) and 90 pmol of each primer (Sangon Biotech Co., Ltd.). The PCR conditions were as follows: Initial denaturation at $95^{\circ} \mathrm{C}$ for $5 \mathrm{~min}$, followed by 35 amplification cycles, with each cycle including a $45 \mathrm{sec}$ denaturation step at $94^{\circ} \mathrm{C}, 1 \mathrm{~min}$ annealing step at a primer specific temperature, and a 1 min elongation step at $72^{\circ} \mathrm{C}$, the process was completed with a final $10 \mathrm{~min}$ extension step at $72^{\circ} \mathrm{C}$. The annealing temperatures for the PCR primer sequences of the selected genes are presented in Table I.

Following PCR amplification, electrophoresis was performed to separate the products, using $2 \%$ agarose gel (Sangon Biotech Co., Ltd.) stained by GeneGreen (Tiangen Biotech Co., Ltd., Beijing, China) nucleic acid dye and the products were detected under ultra violet light. Target products were sequenced by Sangon Biotech Co., Ltd. (Shanghai, China) and the data were confirmed by repeating the PCR amplification and sequence analysis at least twice.

The sequences were subsequently analyzed by NCBI Blast (blast.ncbi.nlm.nih.gov/Blast.cgi) and DNAMAN version 5.2.2 (Lynnon Biosoft LLC, San Ramon, CA, USA). HPV-56 nucleotide positions were numbered according to the reference sequence X74483. Only when the LCR-E6-E7-L1 were amplified and sequenced simultaneously, was the resulting sequence selected for subsequent analyses.

Phylogenetic trees analysis. Phylogenetic trees of HPV-56 LCR-E6-E7 and L1 were constructed with the maximum-likelihood trees method by Molecular Evolutionary Genetics Analysis version 6 software using Kimura's two-parameter model, respectively. The reference viral sequences EF177181, EF177180, EF177179, EF177178, KU298915, KU298916, KU298918, KU298917, KU298919, EF177176, EF177177, collected from the GenBank sequence database were used to construct the distinct phylogenetic branches. The tree topology was evaluated by using bootstrap resampled 1,000 times as previously described (27). Numbers above the branches indicate the bootstrap values that are $>70 \%$.

Sequence analysis. The secondary structure was predicted with the PSIPRED server (bioinf.cs.ucl.ac.uk/psipred/), which provides a simple and accurate secondary structure prediction method as previously described (28). The prediction of the damaging effect of missense mutation to protein structure and function was performed using PolyPhen-2 software (genetics.bwh.harvard.edu/pph2/) (29).

The MATCH 1.0 server (www.gene-regulation.com/pub/ programs.html\#match) (30) was used to search within the 
Table I. Primers used for the molecular characterization of human papillomavirus-56 E6, E7 and L1 long control regions .

\begin{tabular}{lllrcc}
\hline Gene & Direction & \multicolumn{1}{c}{ Sequence 5'-3' } & $\begin{array}{c}\text { Primer } \\
\text { position }\end{array}$ & $\begin{array}{c}\text { Product } \\
\text { size, bp }\end{array}$ & $\begin{array}{c}\text { Annealing } \\
\text { Temperature, }{ }^{\circ} \mathrm{C}\end{array}$ \\
\hline LCR & Forward & TGTGTCATTATTGTGGCTTTTGTTTTGT & 7323 & 603 & 57 \\
& Reverse & AGCTGCCTTTTATATGTACCGTTTC & 81 & & 58 \\
E6 & Forward & ATTGGGAGTGACCGAAAAGG & 26 & 803 & 58 \\
& Reverse & ACAACACGCAGGTCCTCTTT & 828 & & 57.5 \\
\multirow{2}{*}{ L1-1 } & Forward & GCTACAGATGTCAAAGTCCG & 444 & 542 & 59 \\
& Reverse & GCCTCTACTTCAAACCATCC & 985 & & 5 \\
L1-2 & Forward & GCCCCTTTAGGTAATGTGTGG & 5418 & 943 & 58 \\
& Reverse & CCACATAGAATCACCATAGGCAT & 6360 & & 5 \\
& Forward & ATGATAGACACAGGATTTGGCG & 6217 & 948 & \\
\hline
\end{tabular}

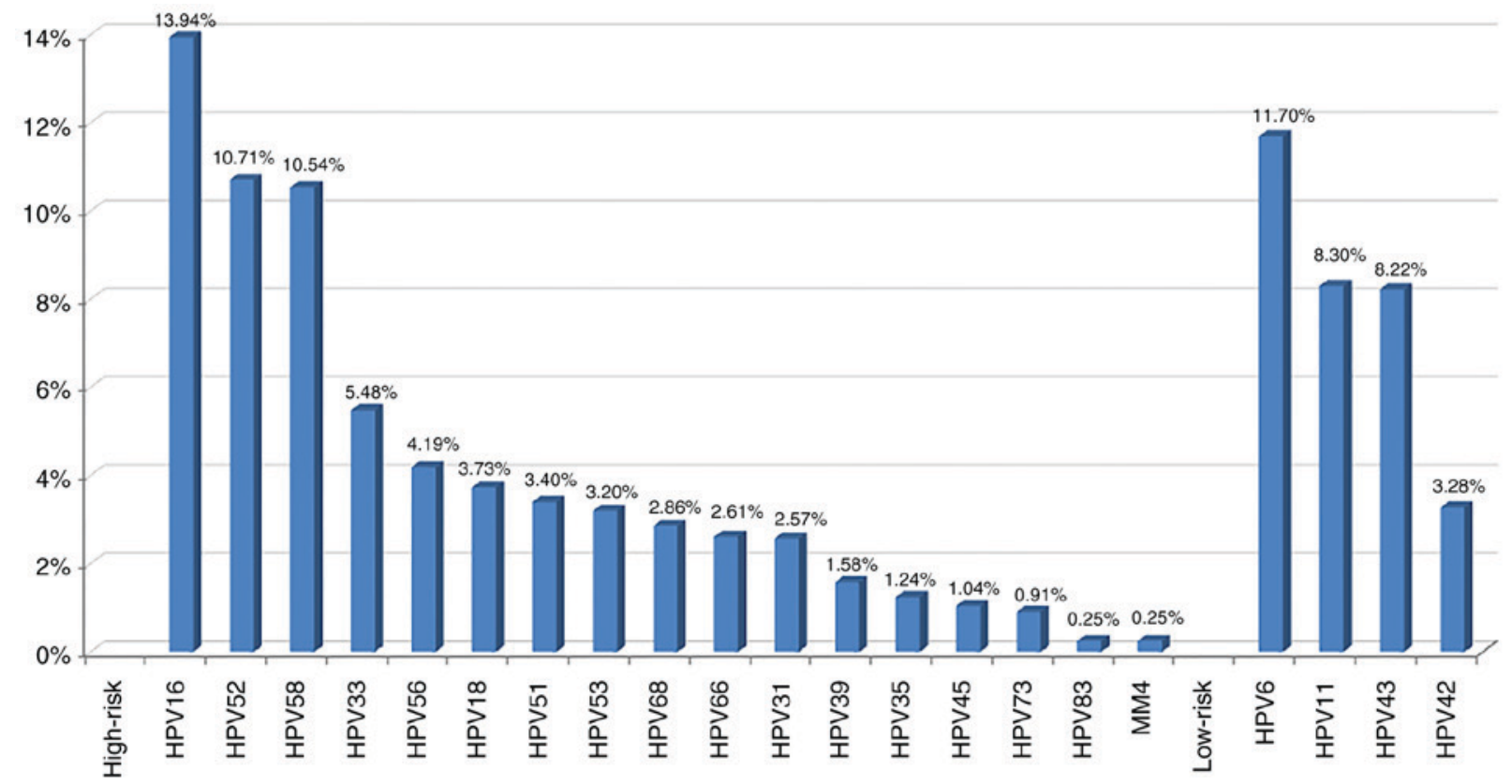

Figure 1. Detection rates of the 23 types of HPV in the 7,707 women sampled. HPV, human papillomavirus.

LCR of HPV-56 for potential binding sites for cellular and viral transcriptional factors. Various transcriptional factors were identified, including AP-1, E2, GRE, NF-1, Oct-1, TATA, YY1, C/EBP, Sp1, SRY, AML-1a and c-Myc/c-Max.

Selective pressure analysis. To estimate for positive selection at particular sites of the HPV-56 E6-E7 and L1 gene sequences, the codeml program in the Phylogenetic Analyses by Maximum Likelihood (PAML) version 4.8 package was used to perform likelihood ratio tests to infer nonsynonymous and synonymous nucleotide divergence for coding regions by the method of Nei and Gojobor (31).

\section{Results}

Characteristics of HPV-56 prevalence in southwest China in the span of 2 years. During the period of January 2014 to January 2016, the overall prevalence of detectable HPV infection was $31.27 \%(2,407 / 7,707)$ and $21.42 \%(1,651 / 7,707)$ samples were high-risk HPV type and 9.85\% (759/7,707) were low-risk HPV type. From the HPV-positive women involved in the present study, the most commonly detected high-risk HPV genotype was HPV-16 (336, 13.94\%), followed by HPV-52 (258, 10.71\%), HPV-58 (254, 10.54\%) and HPV-33 (132, $5.48 \%)$. HPV-56 ranked fifth $(101,4.19 \%)$. Additional high-risk HPV genotypes were listed in descending order of priority as follows: HPV-18, -51, -53, -68, -66, -31, -39, -35, -45, -73, -83 and MM4 (Fig. 1).

HPV-56 LCR-E6-E7 sequence variation. From the 101 samples which tested positive for HPV-56, 75 (75.25\%) LCR-E6-E7-L1 sequences were amplified by PCR for variant analysis. The remaining $26(25.75 \%)$ sequences were not obtained due to unsuccessful PCR. 
Table II. Nucleotide sequence variations at long control regions-E6-E7 of 23 HPV-56 isolates.

\begin{tabular}{|c|c|c|c|c|c|c|c|c|c|c|c|c|c|c|c|c|c|c|c|c|c|c|c|c|c|c|c|c|c|c|c|c|c|c|c|c|c|c|}
\hline \multicolumn{20}{|c|}{ LCR } & \multicolumn{11}{|c|}{ E6 } & \multicolumn{7}{|c|}{ E7 } & \multirow[b]{5}{*}{$\mathrm{n}, 75$} \\
\hline & 7 & 7 & 7 & 7 & & 7 & 7 & 7 & 7 & 7 & 7 & 7 & 7 & 7 & 7 & 7 & 7 & 7 & 7 & & & & & & & & & & & & & & & & & & & \\
\hline & 4 & 4 & 4 & 5 & D & 5 & 5 & 5 & 5 & 5 & 5 & 5 & 6 & 6 & 7 & 7 & 7 & 7 & 8 & 1 & 1 & 2 & 2 & 2 & 2 & 2 & 2 & 3 & 4 & 4 & 6 & 6 & 6 & 7 & 8 & 8 & 8 & \\
\hline & 4 & 6 & 8 & 0 & & 4 & 5 & 8 & 8 & 8 & 9 & 9 & 2 & 7 & 4 & 7 & 8 & 9 & 0 & 4 & 5 & 1 & 5 & 5 & 6 & 6 & 7 & 3 & 3 & 4 & 0 & 0 & 1 & 7 & 0 & 0 & 8 & \\
\hline Isolates & 1 & 9 & 5 & 4 & & 6 & 9 & 1 & 2 & 9 & 3 & 6 & 8 & 8 & 1 & 7 & 1 & 6 & 0 & 1 & 8 & 5 & 3 & 4 & 3 & 4 & 9 & 2 & 7 & 3 & 1 & 5 & 8 & 0 & 0 & 2 & 1 & \\
\hline Ref & $\mathrm{A}$ & G & $T$ & $\mathrm{~T}$ & & G & $T$ & $\mathrm{~T}$ & G & A & G & $\mathrm{T}$ & A & G & A & G & C & $\mathrm{T}$ & $\mathrm{T}$ & A & $T$ & A & C & $\mathrm{T}$ & $\mathrm{A}$ & $\mathrm{T}$ & G & $\mathrm{A}$ & G & $\mathrm{T}$ & $\mathrm{C}$ & G & $\mathrm{C}$ & G & $\mathrm{C}$ & G & A & \\
\hline 56SE01 & - & - & $\mathrm{C}$ & $\mathrm{G}$ & D1 & - & - & $\mathrm{C}$ & - & - & $\mathrm{C}$ & - & $\mathrm{C}$ & - & - & - & - & - & G & - & - & - & - & - & - & - & $\mathrm{A}$ & - & - & - & $\mathrm{A}$ & $\mathrm{A}$ & - & - & - & - & - & 1 \\
\hline 56SE02 & $\cdot$ & - & C & . & D2 & - & - & $\mathrm{C}$ & $\cdot$ & - & A & $\mathrm{C}$ & C & - & - & - & - & - & G & - & - & . & - & - & - & - & A & $\cdot$ & - & - & $\mathrm{A}$ & $\mathrm{A}$ & - & - & - & - & - & 1 \\
\hline 56SE03 & $\mathrm{C}$ & $\mathrm{T}$ & $\mathrm{C}$ & . & D2 & - & c & $\mathrm{C}$ & - & - & A & $\mathrm{C}$ & $\mathrm{C}$ & - & - & - & - & - & G & - & $\mathrm{C}$ & - & - & - & - & - & A & - & A & - & $\mathrm{A}$ & - & - & - & - & - & - & 1 \\
\hline 56SE04 & - & - & $\mathrm{c}$ & . & D2 & - & - & $\mathrm{c}$ & - & - & A & $\mathrm{C}$ & $\mathrm{c}$ & - & - & - & - & - & G & - & $\mathrm{C}$ & - & - & - & - & - & $\mathrm{A}$ & - & A & - & $\mathrm{A}$ & - & - & $\mathrm{C}$ & - & - & - & 1 \\
\hline 56SE05 & - & - & C & . & D2 & - & - & $\mathrm{C}$ & - & - & $\mathrm{A}$ & C & c & - & - & A & - & - & G & - & $\mathrm{C}$ & - & - & - & - & - & $\mathrm{A}$ & - & A & - & $\mathrm{A}$ & - & - & - & - & - & - & 1 \\
\hline 56SE06 & - & - & C & . & D2 & - & - & $\mathrm{C}$ & - & - & A & $\mathrm{C}$ & $\mathrm{C}$ & - & - & - & - & - & G & - & $\mathrm{C}$ & - & - & - & - & - & A & G & A & - & $\mathrm{A}$ & - & - & $\mathrm{C}$ & - & $\mathrm{C}$ & - & 1 \\
\hline 56SE07 & $\cdot$ & - & $\mathrm{C}$ & . & D2 & - & - & $\mathrm{C}$ & - & - & A & $\mathrm{C}$ & $\mathrm{C}$ & - & - & - & - & - & G & - & $\mathrm{C}$ & - & - & - & - & - & A & G & A & - & $\mathrm{A}$ & - & - & $\mathrm{C}$ & $\cdot$ & . & - & 3 \\
\hline 56SE08 & - & - & $\mathrm{c}$ & . & D2 & - & - & $\mathrm{c}$ & - & G & $\mathrm{A}$ & $\mathrm{c}$ & $\mathrm{c}$ & - & - & - & - & - & G & - & $\mathrm{C}$ & - & - & - & - & - & $\mathrm{A}$ & - & A & - & $\mathrm{A}$ & - & - & $\mathrm{C}$ & - & - & - & 1 \\
\hline 56SE09 & - & - & $\mathrm{C}$ & . & D2 & - & - & $\mathrm{C}$ & - & - & A & $\mathrm{C}$ & $\mathrm{c}$ & - & - & - & - & - & G & - & $\mathrm{c}$ & - & - & - & - & - & A & - & A & - & $\mathrm{A}$ & - & - & $\mathrm{C}$ & - & - & - & 17 \\
\hline 56SE10 & $\mathrm{C}$ & $\mathrm{T}$ & $\mathrm{C}$ & . & D2 & - & $\mathrm{C}$ & $\mathrm{C}$ & - & - & A & C & $\mathrm{C}$ & - & - & - & - & - & G & - & $\mathrm{C}$ & - & - & - & - & - & $\mathrm{A}$ & - & A & - & - & $\mathrm{A}$ & - & - & - & $\mathrm{C}$ & - & 1 \\
\hline 56SE11 & - & $\mathrm{T}$ & C & . & D2 & - & $\mathrm{C}$ & $\mathrm{C}$ & - & - & - & $\mathrm{C}$ & $\mathrm{C}$ & - & - & - & - & - & G & $\mathrm{C}$ & - & - & - & - & C & - & - & - & - & - & - & $\mathrm{A}$ & - & - & - & $\mathrm{C}$ & - & 11 \\
\hline 56SE12 & - & $\mathrm{T}$ & $\mathrm{C}$ & . & D2 & $\mathrm{A}$ & c & $\mathrm{C}$ & - & - & $\cdot$ & C & $\mathrm{C}$ & $\cdot$ & - & - & A & - & G & $\mathrm{C}$ & $\cdot$ & G & $\mathrm{T}$ & C & $\mathrm{C}$ & - & - & - & - & - & - & $\mathrm{A}$ & - & - & - & $\mathrm{C}$ & - & 1 \\
\hline 56SE13 & - & $\mathrm{T}$ & $\mathrm{C}$ & . & D2 & - & - & $\mathrm{C}$ & - & - & - & $\mathrm{C}$ & $\mathrm{C}$ & - & - & - & - & - & G & $\mathrm{C}$ & - & - & - & - & $\mathrm{C}$ & - & - & - & $\mathrm{A}$ & - & - & $\mathrm{A}$ & - & - & $\mathrm{T}$ & $\mathrm{C}$ & - & 1 \\
\hline 56SE14 & $\mathrm{C}$ & $\mathrm{T}$ & $\mathrm{C}$ & . & D2 & - & $\mathrm{c}$ & $\mathrm{C}$ & - & - & $\cdot$ & $\mathrm{C}$ & $\mathrm{C}$ & - & - & - & - & - & G & $\mathrm{C}$ & - & - & - & - & $\mathrm{C}$ & - & - & - & - & - & - & $\mathrm{A}$ & - & - & - & $\mathrm{C}$ & - & 2 \\
\hline 56SE15 & $\mathrm{c}$ & $\mathrm{T}$ & $\mathrm{C}$ & . & D2 & - & $\mathrm{c}$ & $\mathrm{c}$ & A & - & $\mathrm{A}$ & $\mathrm{C}$ & $\mathrm{c}$ & - & - & - & - & - & G & $\mathrm{C}$ & - & - & - & - & $\mathrm{C}$ & - & - & - & - & - & - & $\mathrm{A}$ & - & - & - & $\mathrm{c}$ & - & 1 \\
\hline 56SE16 & $\mathrm{C}$ & $\mathrm{T}$ & $\mathrm{C}$ & . & D2 & - & $\mathrm{C}$ & $\mathrm{c}$ & - & - & $\mathrm{A}$ & C & $\mathrm{C}$ & - & - & - & - & - & G & $\mathrm{C}$ & - & - & - & - & $\mathrm{C}$ & - & - & - & - & - & - & $\mathrm{A}$ & $\mathrm{A}$ & - & - & $\mathrm{C}$ & - & 1 \\
\hline $56 \mathrm{SE} 17$ & $\mathrm{C}$ & $\mathrm{T}$ & C & . & D2 & - & $\mathrm{C}$ & $\mathrm{C}$ & - & - & A & $\mathrm{C}$ & $\mathrm{C}$ & - & - & - & - & - & G & $\mathrm{C}$ & - & $\cdot$ & - & $\cdot$ & $\mathrm{C}$ & G & $\cdot$ & - & - & - & - & $\mathrm{A}$ & - & $\cdot$ & $\cdot$ & $\mathrm{C}$ & - & 1 \\
\hline 56SE18 & $\mathrm{C}$ & $\mathrm{T}$ & C & . & D2 & - & C & $\mathrm{C}$ & - & - & A & $\mathrm{C}$ & C & $\cdot$ & - & - & - & - & G & $\mathrm{C}$ & - & - & - & - & $\mathrm{C}$ & - & - & - & - & A & - & $\mathrm{A}$ & - & - & - & $\mathrm{C}$ & - & 24 \\
\hline 56SE19 & $\mathrm{c}$ & $\mathrm{T}$ & $\mathrm{c}$ & . & D2 & - & $\mathrm{c}$ & $\mathrm{c}$ & - & - & $\mathrm{A}$ & $\mathrm{c}$ & $\mathrm{c}$ & - & c & - & - & - & G & $\mathrm{c}$ & - & - & - & - & $\mathrm{c}$ & - & - & - & - & A & - & $\mathrm{A}$ & - & - & - & $\mathrm{c}$ & - & 1 \\
\hline 56SE20 & $\mathrm{C}$ & $\mathrm{T}$ & C & . & D2 & - & $\mathrm{c}$ & $\mathrm{C}$ & - & - & $\mathrm{A}$ & $\mathrm{C}$ & $\mathrm{c}$ & - & - & - & - & - & G & $\mathrm{c}$ & - & - & - & - & $\mathrm{C}$ & - & - & - & - & A & - & $\mathrm{A}$ & - & - & - & $\mathrm{c}$ & $\mathrm{C}$ & 1 \\
\hline 56SE21 & $\mathrm{C}$ & $\mathrm{T}$ & $\mathrm{C}$ & . & D2 & - & $\mathrm{C}$ & $\mathrm{C}$ & - & - & A & $\mathrm{C}$ & $\mathrm{C}$ & - & - & - & - & G & G & $\mathrm{C}$ & - & - & - & - & $\mathrm{C}$ & - & $\cdot$ & - & - & A & - & $\mathrm{A}$ & - & - & $\cdot$ & $\mathrm{C}$ & - & 1 \\
\hline 56SE22 & $\mathrm{C}$ & $\mathrm{T}$ & $\mathrm{C}$ & . & D2 & - & $\mathrm{c}$ & $\mathrm{C}$ & - & $\cdot$ & A & $\mathrm{C}$ & $\mathrm{C}$ & A & - & - & - & - & G & $\mathrm{C}$ & - & - & - & - & $\mathrm{C}$ & - & $\cdot$ & - & - & A & - & $\mathrm{A}$ & - & - & $\cdot$ & $\mathrm{c}$ & - & 1 \\
\hline 56SE23 & $\mathrm{C}$ & $\mathrm{T}$ & $\mathrm{C}$ & . & D2 & - & $\mathrm{C}$ & $\mathrm{C}$ & - & - & A & $\mathrm{C}$ & $\mathrm{C}$ & - & - & - & - & - & G & $\mathrm{C}$ & - & - & - & - & $\mathrm{C}$ & - & - & - & - & A & - & $\mathrm{A}$ & - & - & $\mathrm{T}$ & $\mathrm{C}$ & - & 1 \\
\hline & $\bar{\nearrow}$ & $\bar{\nearrow}$ & & - & $\bar{\nearrow}$ & - & 它 & $\begin{array}{l}\grave{2} \\
\text { 心 } \\
\vdots \\
\vdots \\
\vdots\end{array}$ & 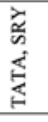 & 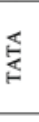 & - & - & - & - & - & 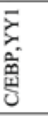 & $\succsim$ & $\bar{\nearrow}$ & $\bar{\nearrow}$ & $\frac{a}{n}$ & - & - & 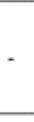 & $\overrightarrow{\bar{n}}$ & $\begin{array}{l}\text { z } \\
\stackrel{\text { Z }}{2}\end{array}$ & 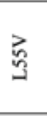 & z్ & - & - & - & 띵 & $\stackrel{\bar{y}}{>}$ & $\stackrel{ }{6}$ & 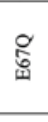 & 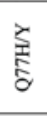 & $\underset{\sigma}{\stackrel{5}{E}}$ & $\frac{\frac{o}{d}}{n}$ & \\
\hline $\begin{array}{l}\text { Second } \\
\text { structure }\end{array}$ & & & & & & & & & & & & & & & & & & & & - & $\mathrm{H}$ & - & - & - & $\mathrm{s}$ & $\mathrm{s}$ & - & $\mathrm{H}$ & - & $\mathrm{H}$ & - & $\mathrm{s}$ & - & - & $\mathrm{s}$ & $\mathrm{s}$ & - & \\
\hline
\end{tabular}

$(-)$, conserved nucleotides with respect to the reference sequence; (.), a nucleotide deletion. Letters indicate variation in position. D1, deletion of 19 bp (7,509-7,527) among HPV-56 isolates; D2, deletion of 42 bp (7,492-7,533). S, Sheet; H, Helix; HPV, human papillomavirus.

All 75 HPV-56 LCR-E6-E7 isolates showed nucleotide variation when compared with the HPV-56 reference sequence (GenBank: X74483). The isolates were divided into 23 different groups denoted as 56SE01-56SE23. And these sequences were published with GenBank accession codes from KX645742 to KX645764.

From the HPV-56 LCR sequences, 18 point substitutions were detected, 11 of which were located in putative transcriptional factors binding sites and four substitutions (T7485C, T7581C, A7628C and T7800G) were present in all isolates. In addition, a 42 bp deletion and a 19 bp deletion were observed, leading to loss of a putative binding site for the YY1 transcriptional factor (Table II).

A total of 18 single nucleotide changes occurred in the E6-E7 sequence, with 11/18 (61.11\%) being non-synonymous substitutions and 7/18 (38.89\%) being synonymous mutations. The sequence variability of E6 was lower than that of E7. The average probability of a nucleotide sequence deviation from the prototype was 6.2 substitutions per 1,000 bp for E6 and 6.4 substitutions per 1,000 bp for E7 (Table II). Three amino acid mutations occurred in sequences encoding the $\alpha$-helix, and five mutations (four non-synonymous) were observed in sequences encoding the $\beta$-sheet (Table II). Additionally, PolyPhen-2 analysis predicted a $\mathrm{Q} 77 \mathrm{H} / \mathrm{Y}$ mutation of E7 to be "possibly damaging' with a score of $0.702 / 0.610$ on the HumVar model (Fig. 2). Mutations generating a frame shift or a premature stop codon were not observed.

Phylogenetic analysis of HPV-56 LCR-E6-E7 sequences. Phylogenetic analysis based on HPV-56 LCR-E6-E7 1,259 bp nucleotide sequences was performed including 23 isolates in the current study, accompanied by 12 reference sequences. Based on whole genome study and the topology, two distinct branches were formed (Fig. 3) (32). A total of 9 isolates in the current study (56SE01-S6SE09) were classified into the A variant lineage. The remaining 14 isolates (56SE10-S6SE23) were classified into the B variant lineage.

HPV-56 L1 sequence variation. The nucleotide variation rate of HPV-56 L1 was $100 \%$ among the 75 HPV-56 isolates when compared with the HPV-56 reference sequence (GenBank: $\mathrm{X} 74483)$. The $\mathrm{L} 1$ isolates were divided into 16 different groups denoted as 56HL01-56HL16 (Table III). And these sequences were published in GenBank with the accession codes KX645765-645780.

A total of 24 single nucleotide changes were identified in the 1,605 bp L1 sequence, $6(33.33 \%)$ substitutions were non-synonymous mutations and 18 (66.67\%) substitutions 
A

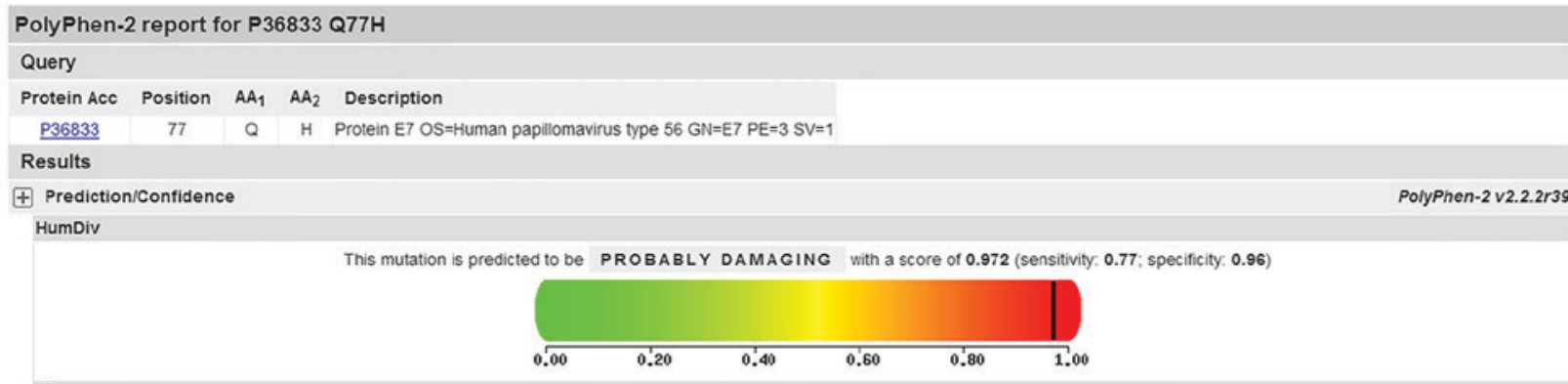

$\bigoplus$ HumVar

This mutation is predicted to be POS SIBLY DAMAGING wth a score of 0.702 (sensitivity. 0.78 ; specificity 0.85

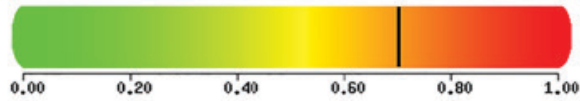

B

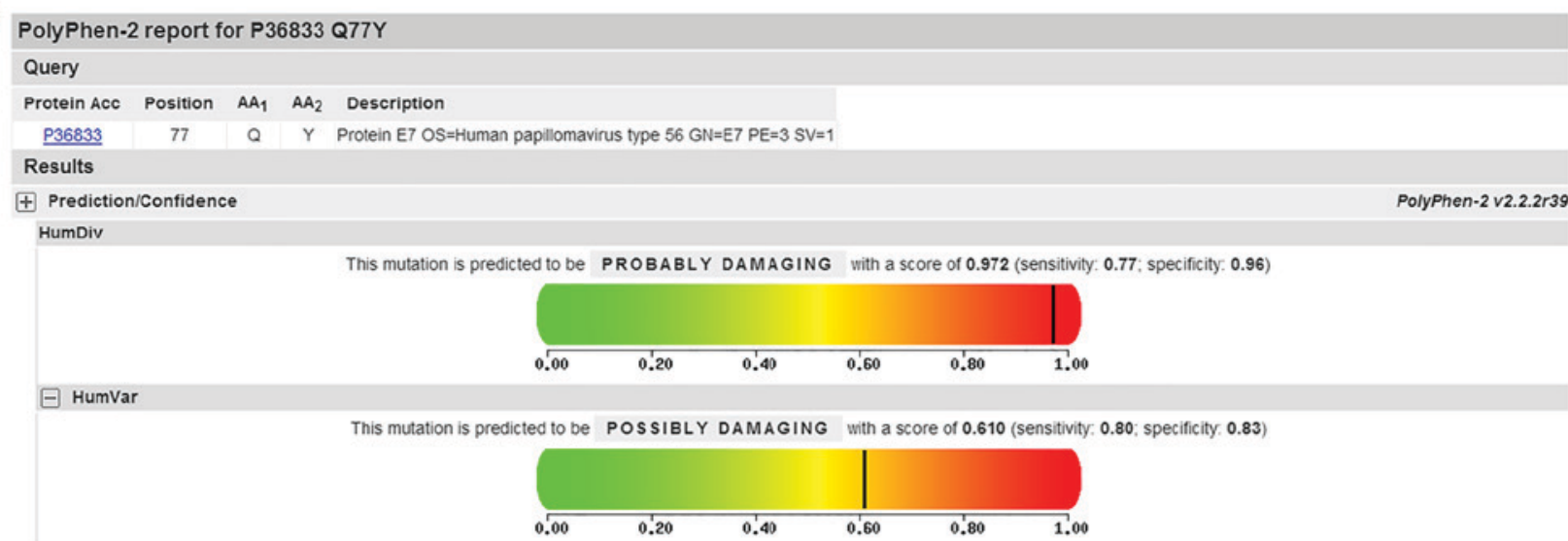

Figure 2. Predicted of HPV-56 E7 Q77H/Y substitution the pathogenicity by PolyPhen-2. Pathogenicity of (A) HPV-56 E7 Q77H and (B) HPV-56 E7 Q77Y substitution. HPV, human papillomavirus.

were synonymous mutations and G64554T was present in all samples analyzed (Table III). The average probability of a nucleotide sequence deviation from prototype was 4.04 substitutions per 1,000 bp for L1. No non-synonymous mutation was observed in the HPV-56 L1 sequences encoding the a-helix. 8 mutations (one non-synonymous) were observed in the sequences encoding the $b$ sheet (Table III). In addition, PolyPhen-2 analysis predicted that K116M and N126D mutations of L1 were 'probably damaging' on HumVar model with the score of 0.975 and 0.918 , respectively (Fig. 4). No frame shift and premature stop caused by mutations were observed.

Phylogenetic analysis of HPV-56 L1 sequences. Phylogenetic analysis based on HPV-56 L1 1,605 bp nucleotide sequences was performed including 16 isolates in the current study, accompanied by 12 reference sequences. Based on whole genome study and the topology, 2 distinct branches were formed (Fig. 5) (32). A total of 8 isolates in the current study (56HL01-S6HL08) were classified into the A variant lineage. The remaining 8 isolates (56HL09-S6HL18) were classified into the $\mathrm{B}$ variant lineage.

Selective pressure analysis of HPV 56 E6-E7 and L1 sequences. PAML software was used to estimate the selective pressure of HPV-56. Positive selection for E6-E7 and L1 was identified and has been presented in Tables IV and V, respectively. In addition, there was no evidence of negative selection in the sequence alignment of these genes $(\mathrm{P}<0.1)$.

\section{Discussion}

When considering the association between HPV and cervical cancer, HPV-16 and HPV-18 are the most frequent worldwide, with HPV-56 only accounting for a small proportion of the infections worldwide, with no available commercial vaccines (33). However, the prevalence of high-risk HPV types differs greatly among different countries and regions. Previous studies have revealed a high detection rate of the HPV-56 strain among Chinese women (9-11). The purpose of the present study was to determine a ranking of HPV type prevalence and to demonstrate the importance and significance of HPV-56 in southwest China.

Previous studies have demonstrated that specific intratypic HPV genome variations may affect the virus infectivity, pathogenicity, oncogenicity, viral particle assembly and host immune response $(15,34)$. In the current study, the genetic variability of LCR, E6, E7 and L1 genes of HPV-56 in southwest China were analyzed. Additionally, phylogenetic trees were constructed for all variant strains and selection pressures of the E6, E7, and L1 genes were estimated.

The binding affinity of the cellular and viral transcriptional factor may be influenced by nucleotide variation within LCR (35). Some of the mutations identified in the LCR in the 


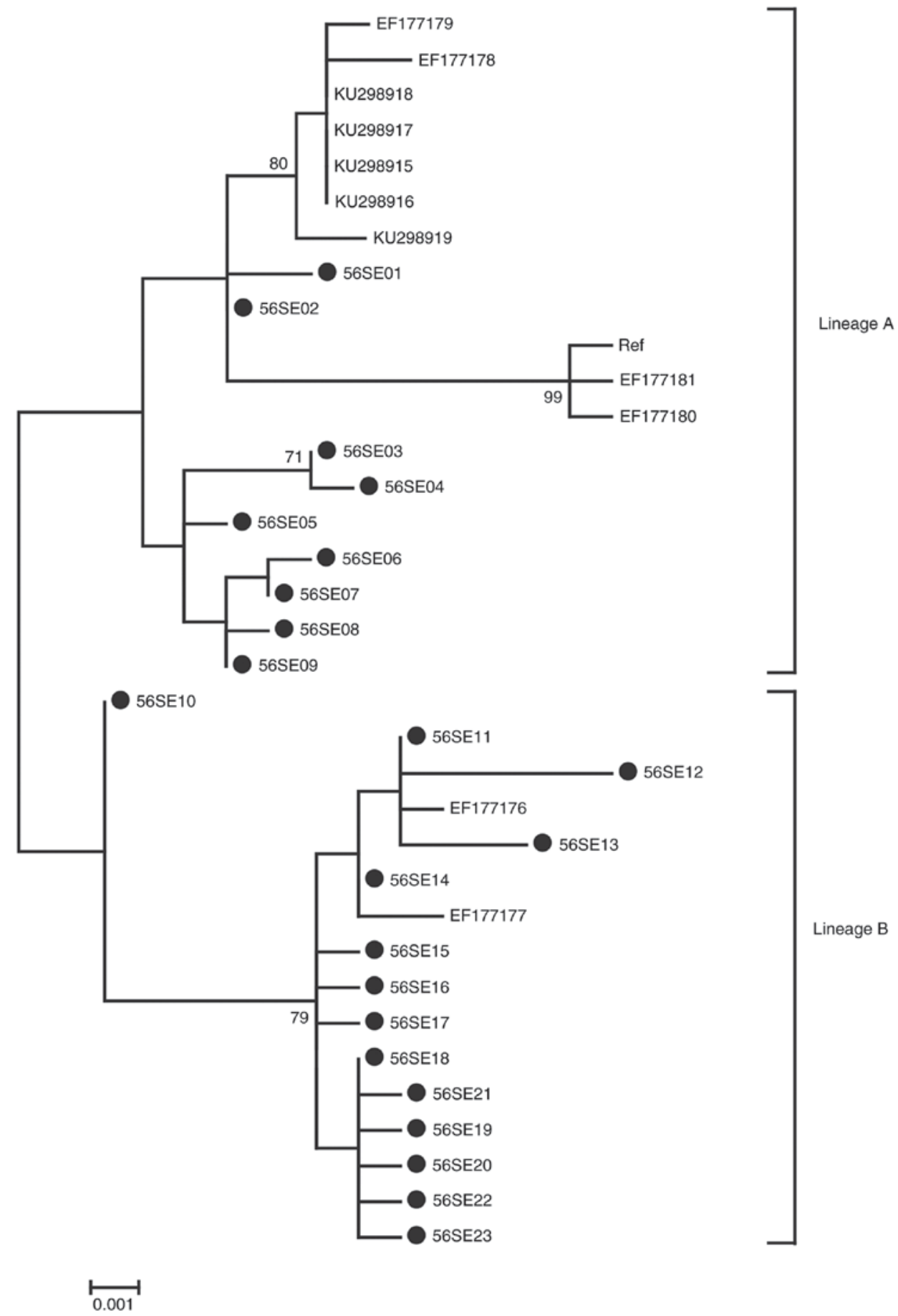

Figure 3. Maximum-likelihood trees of LCR-E6-E7 of HPV-56. The maximum-likelihood tree of HPV-56 variants based on LCR-E6-E7 combined sequences Sequences from the present study are labeled in black, the remaining are standard sequences used for alignment. The numbers above the branches indicate the bootstrap values $>70 \%$.

present study are embedded in the putative binding sites for YY1, SRY,NF-1, TATA and C/EBP transcription factors. These mutations may affect the binding affinity of transcriptional factor and the transcription of the downstream E6 and E7 oncogenes; however, it remains to be determined by in vitro studies. When compared with a previous study, changes at positions 7,485 , 7,504, 7,546, 7,582, 7,589, 7,678, 7,741, 7,781 and 7,796 of LCR, to the best of our knowledge, were described for the first time in the present study, and a new 19 bp deletion was not described previously (14).
The early expressing proteins E6 and E7 of high-risk HPV are the primary oncoproteins involved in human epithelial cell immortalization and transformation due to their ability to inactivate p53 and pRb proteins, respectively $(36,37)$. In addition, amino acid mutation of E6/E7 genes may influence host immunologic responses (38). Therefore, studies on the genetic variability in HPV E6/E7 may provide important basic data for future research on viral molecular mechanisms and contribute to the design of therapeutic vaccines, which target these proteins (39). In the current study, 11/18 amino acid 


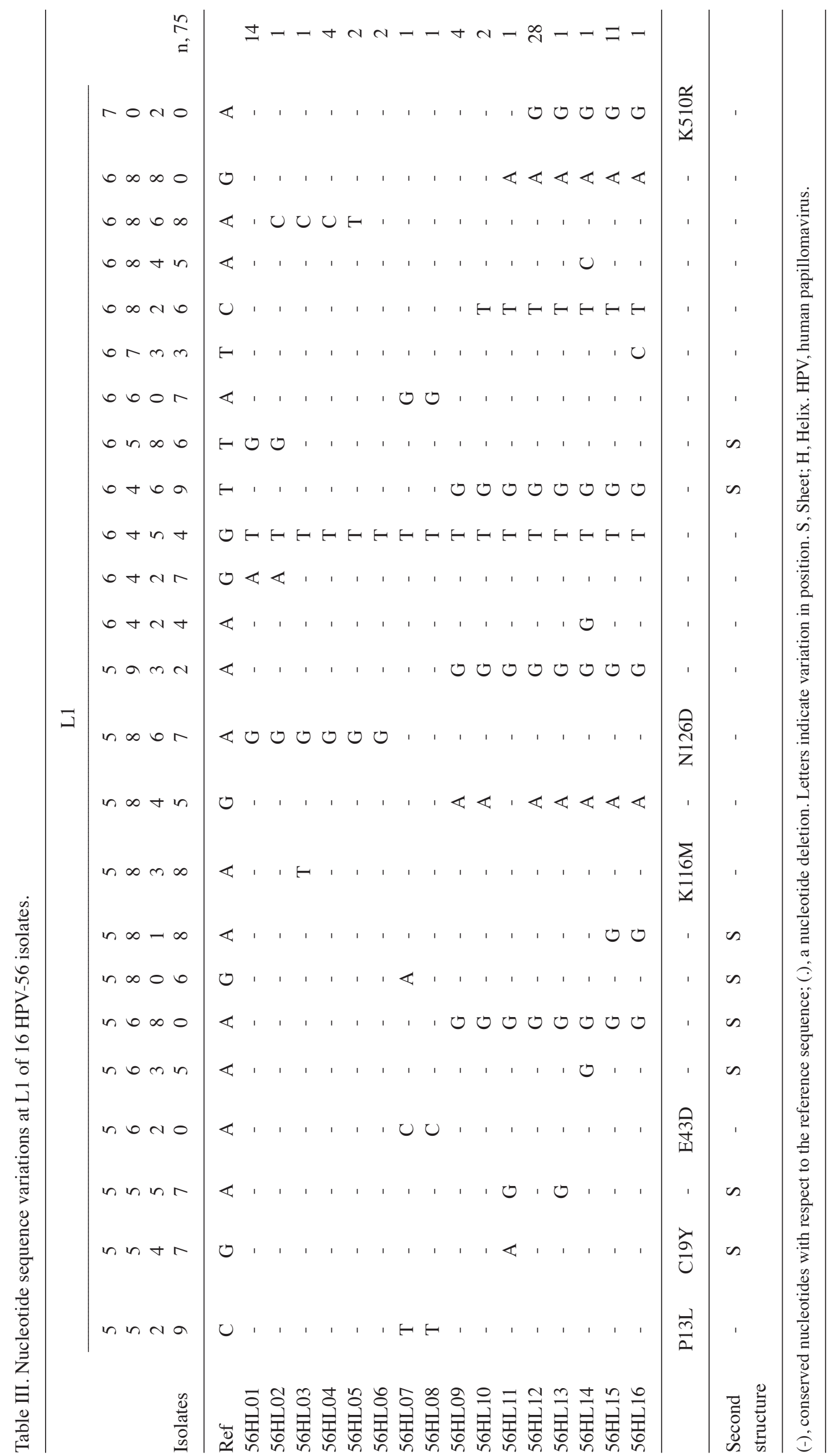




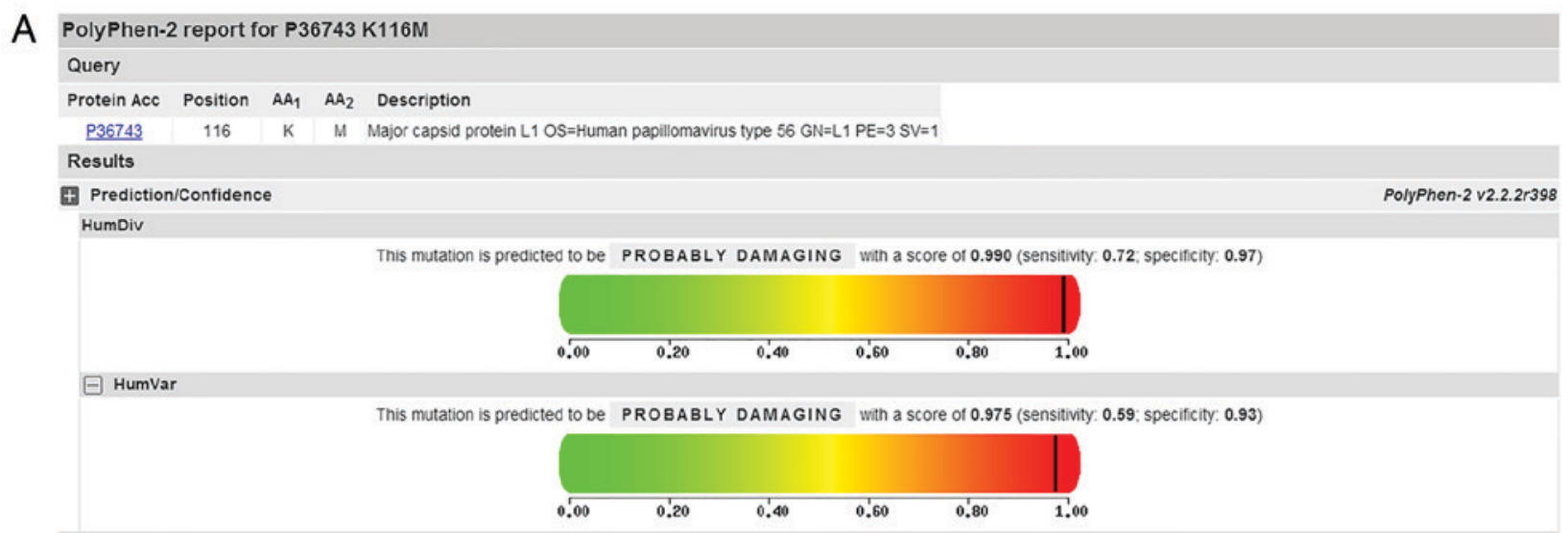

B Polyphen-2 report for P36743 N126D

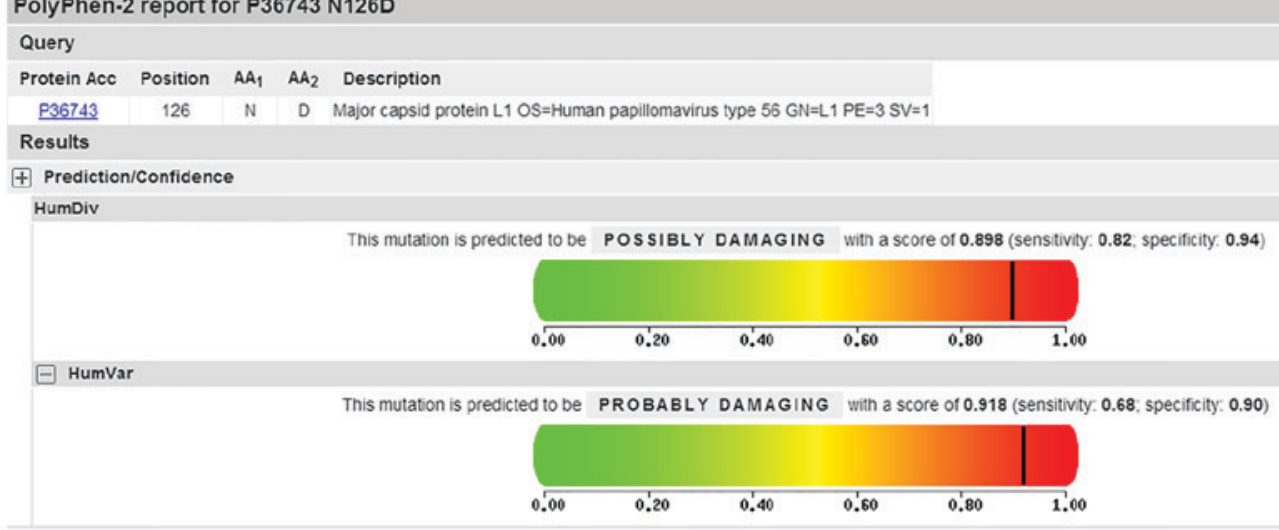

Figure 4. Predicted of HPV-56 L1 K116M and N126D substitutions the pathogenicity by PolyPhen-2. The pathogenicity of (A) HPV-56 L1 K116M and (B) HPV-56 L1 N126D substitution. HPV, human papillomavirus.

mutations identified in E6/E7 were non-synonymous, except for A141C, T158C and A263C, all the remaining mutations were described, to the best of our knowledge, for the first time in the current study. S14R and K54N of E6 amino acid substitutions were apparently associated and were simultaneously found in 47 isolates. It is of note that the present study identified that the E7 variation frequency is higher than E6 variations, similar to findings in the HPV-58 type (40). Variants of the Q77H/Y changes of E7 was analyzed using PolyPhen-2 with results of 'possibly damaging', at the same time, the variant occurred in the E7 $\beta$-sheet, which may influence E7 structure.

Overall, the L1 major capsid protein may be used to inform the design of prophylactic vaccines and main components virus-like particles (VLPs) that induce high levels of neutralizing antibodies (19). Additionally, conformational epitopes of HPV are primarily located in hypervariable immuno-dominant regions (BC, DE, EF, FG and HI loops). Therefore, previous studies demonstrated that polymorphisms in the L1 gene may have a critical role in the structure of the capsid protein, VLPs assembly and recognition epitopes (41-43). The nucleotide changes detected at positions 6,826 and 6,880 of HPV-56 L1 gene were previously described (25); however, to the best of our knowledge the remaining mutations at other positions are described for the first time in the present study. Variants of K116M and N126D were analyzed using PolyPhen-2 and the mutation was identified as 'probably damaging'. Additionally, $\mathrm{C} 19 \mathrm{Y}$ is located in the sequences encoding the b sheet, those variants may affect L1 structure.
In terms of phylogenetics, the current known HPV types have high diversity. According to the maximum-likelihood trees topology constructed by the current study and the reference strains, the strains are distributed in two clusters, not in one single cluster (32). Selective pressure analysis revealed that the majority of the identified HPV amino acid changes were of positive selection, which indicated that these mutations were beneficial for HPV to accommodate the environment.

In summary, the current study reported the prevalence of HPV-56 and sequence variations in the LCR, E6, E7 and L1 genes of HPV-56 isolates from southwest China. Some of the variations identified in LCR are located within binding sites of transcriptional factors. In addition, the majority of nucleotide changes were described for the first time by the current study. To the best of our knowledge, this was the first comprehensive study to assess genetic variation of HPV-56 in southwest China. The present study provided important basic data for further research on viral molecular mechanisms and future development of diagnostic probes and therapeutic vaccines.

\section{Acknowledgements}

The authors would like to thank the following hospitals for the sample collection: Sichuan Reproductive Health Research Center Affiliated Hospital, The Angel Women's and Children's Hospital, The Chengdu Western Hospital Maternity Unit, The Peoples' Hospital of Peng Zhou, Jinjiang Maternity and Child 
Table IV. Site-specific tests for positive selection on human papillomavirus-56 E6-E7.

\begin{tabular}{|c|c|c|c|c|c|}
\hline Models & InL & Parameters estimates & $2 \Delta 1$ & E6 positively selected sites & E7 positively selected sites \\
\hline M7 & -1340.45 & $\mathrm{p}=0.005 ; \mathrm{q}=0.05$ & $\mathrm{n} / \mathrm{a}$ & NA & NA \\
\hline M8 & -1318.69 & $\begin{array}{c}\mathrm{p}_{0}=0.9 ; \mathrm{p}=2.24 ; \mathrm{q}=99 \\
\quad\left(\mathrm{p}_{1}=0.05\right) ; \omega=15.63\end{array}$ & $43.52 ; \mathrm{P}<0.01$ & $14 \mathrm{~S}^{\mathrm{b}} ; 54 \mathrm{~K}^{\mathrm{b}} ; 60 \mathrm{D}^{\mathrm{b}}$ & $10 \mathrm{D}^{\mathrm{b}} ; 12 \mathrm{~V}^{\mathrm{a}} ; 67 \mathrm{E}^{\mathrm{a}} ; 77 \mathrm{Q}^{\mathrm{b}}$ \\
\hline
\end{tabular}

$\operatorname{lnL}, \log$-likelihood difference between the two models; $2 \Delta 1$, twice the log-likelihood difference between the two models; the positively selected

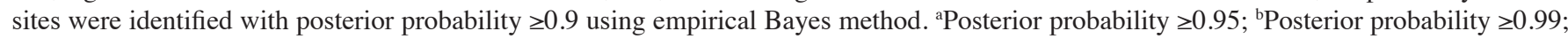
NA, not allowed.

Table V. Site-specific tests for positive selection on human papillomavirus-56 L1.

\begin{tabular}{lcccc}
\hline Models & InL & Estimates of parameters & $2 \Delta 1$ & L1 positively selected sites \\
\hline M7 & -2542.50 & $\mathrm{p}=0.005 ; \mathrm{q}=0.049$ & $\mathrm{n} / \mathrm{a}$ & $\mathrm{NA}$ \\
M8 & -2531.74 & $\mathrm{p}_{0}=0.986 ; \mathrm{p}=0.005 ; \mathrm{q}=4.034 ;\left(\mathrm{p}_{1}=0.014\right) ; \omega=10.537$ & $21.51 ; \mathrm{P}<0.01$ & $510 \mathrm{~K}^{\mathrm{a}}$ \\
\hline
\end{tabular}

$\operatorname{lnL}, \log$-likelihood difference between the two models; $2 \Delta 1$, twice the log-likelihood difference between the two models; the positively selected

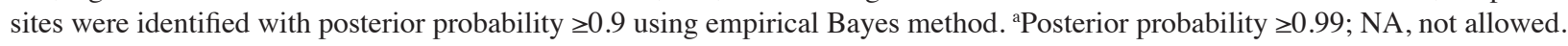

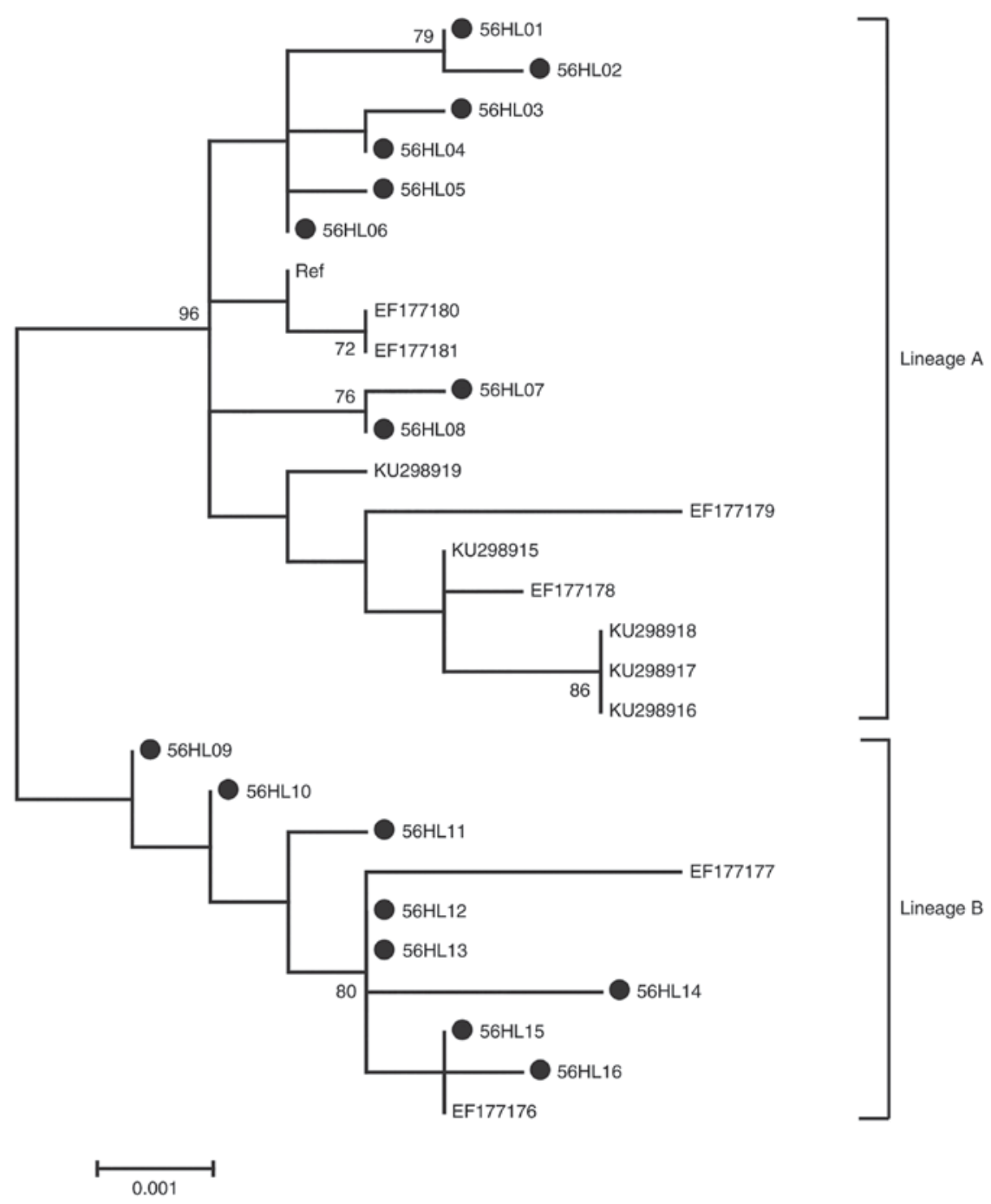

Figure 5. Maximum-likelihood trees of L1 of HPV-56. The maximum-likelihood tree of HPV-56 variants based on L1 sequences. Sequences from the present study are labeled in black, the remaining are standard sequences used for alignment. The numbers above the branches indicate the bootstrap values $>70 \%$. 
Health Hospital, Chengdu Zongnan Gynecology Hospital and Chengdu Songziniao Sterility Hospital.

\section{Funding}

The present study was funded by the Institute of Medical Genetics, College of Life Science, Sichuan University, Chengdu, China.

\section{Availability of data and materials}

All data generated or analysed during this study are included in this published article and GeneBank.

\section{Authors' contributions}

YJ and XD conceived and designed the study. YJ, TW, ZC, JX, XM, MC and HC performed the experiments. ZC and JX analyzed the data. YJ and TW were major contributors in writing the manuscript. All authors read and approved the final manuscript.

\section{Ethics approval and consent to participate}

The present study was approved by the Education and Research Committee and the Ethics Committee of Sichuan University (Chengdu, China; approval number SCU20100196494).

\section{Consent for publication}

Prior to sample collection, written informed consent was obtained from the patients or their guardians, and patient/study subject privacy was carefully protected.

\section{Competing interests}

The authors declare that they have no competing interests.

\section{References}

1. de Sanjose S, Quint WG, Alemany L, Geraets DT, Klaustermeier JE, Lloveras B, Tous S, Felix A, Bravo LE, Shin HR, et al: Human papillomavirus genotype attribution in invasive cervical cancer: A retrospective cross-sectional worldwide study. Lancet Oncol 11: 1048-1056, 2010.

2. Ferlay J SI, Ervik M, Dikshit R, Eser S, Mathers C, Rebelo M, Parkin DM, Forman D and Bray F: GLOBOCAN 2012 v1.0, Cancer Incidence and Mortality Worldwide: IARC CancerBase No. 11. IARC, Lyon, 2013. http://publications.iarc.fr/Databases/ Iarc-Cancerbases/Globocan-2012-Estimated-Cancer-IncidenceMortality-And-Prevalence-Worldwide-In-2012-V1-0-2012.Accessed May 30, 2012.

3. Rampias T, Sasaki C and Psyrri A: Molecular mechanisms of HPV induced carcinogenesis in head and neck. Oral Oncol 50: 356-363, 2014.

4. Bernard HU, Burk RD, Chen Z, van Doorslaer K, zur Hausen H and de Villiers EM: Classification of papillomaviruses (PVs) based on $189 \mathrm{PV}$ types and proposal of taxonomic amendments. Virology 401: 70-79, 2010.

5. Bouvard V, Baan R, Straif K, Grosse Y, Secretan B, El Ghissassi F, Benbrahim-Tallaa L, Guha N, Freeman C, Galichet L, et al: A review of human carcinogens-Part B: Biological agents. Lancet Oncol 10: 321-322, 2009.

6. Arbyn M, Tommasino M, Depuydt C and Dillner J: Are 20 human papillomavirus types causing cervical cancer? J Pathol 234: 431-435, 2014
7. Lörincz AT, Quinn AP, Goldsborough MD, McAllister P and Temple GF: Human papillomavirus type 56: A new virus detected in cervical cancers. J Gen Virol 70: 3099-3104, 1989.

8. Zhai L and Tumban E: Gardasil-9: A global survey of projected efficacy. Antiviral Res 130: 101-109, 2016.

9. Yang L, Yang H, Wu K, Shi X, Ma S and Sun Q: Prevalence of HPV and variation of HPV 16/HPV 18 E6/E7 genes in cervical cancer in women in South West China. J Med Virol 86: 1926-1936, 2014

10. Zeng Z, Yang H, Li Z, He X, Griffith CC, Chen X, Guo X, Zheng B, Wu S and Zhao C: Prevalence and genotype distribution of HPV infection in China: Analysis of 51,345 HPV genotyping results from China's largest CAP certified laboratory. J Cancer 7: 1037-1043, 2016.

11. Wang R, Guo XL, Wisman GB, Schuuring E, Wang WF, Zeng ZY, Zhu $\mathrm{H}$ and Wu SW: Nationwide prevalence of human papillomavirus infection and viral genotype distribution in 37 cities in China. BMC Infect Dis 15: 257, 2015.

12. Chen $Z$, Wang $Q$, Ding $X$, Li Q, Zhong $R$ and Ren $H$ : Characteristics of HPV prevalence in Sichuan Province, China. Int J Gynecol Obstet 131: 277-280, 2015.

13. Lee K, Magalhaes I, Clavel C, Briolat J, Birembaut $\mathrm{P}$, Tommasino $\mathrm{M}$ and Zehbe I: Human papillomavirus 16 E6, L1, L2 and E2 gene variants in cervical lesion progression. Virus Res 131: 106-110, 2008.

14. Cento V, Rahmatalla N, Ciccozzi M, Perno CF and Ciotti M: Intratype variations of HPV 31 and 58 in Italian women with abnormal cervical cytology. J Med Virol 83: 1752-1761, 2011.

15. Xi LF, Schiffman M, Koutsky LA, Hughes JP, Hulbert A, Shen Z, Galloway DA and Kiviat NB: Variant-specific persistence of infections with human papillomavirus Types 31, 33, 45, 56 and 58 and risk of cervical intraepithelial neoplasia. Int J Cancer 139: 1098-1105, 2016.

16. Hubert WG: Variant upstream regulatory region sequences differentially regulate human papillomavirus type 16 DNA replication throughout the viral life cycle. J Virol 79: 5914-5922, 2005.

17. Vande Pol SB and Klingelhutz AJ: Papillomavirus E6 oncoproteins. Virology 445: 115-137, 2013.

18. Roman A and Munger K: The papillomavirus E7 proteins. Virology 445: 138-168, 2013.

19. Buck CB, Day PM and Trus BL: The papillomavirus major capsid protein L1. Virology 445: 169-174, 2013.

20. Chan PK, Luk AC, Park JS, Smith-McCune KK, Palefsky JM, Konno R, Giovannelli L, Coutlée F, Hibbitts S, Chu TY, et al: Identification of human papillomavirus type 58 lineages and the distribution worldwide. J Infect Dis 203: 1565-1573, 2011.

21. Sun Z, Lu Z, Liu J, Wang G, Zhou W, Yang L, Liu C and Ruan Q: Genomic polymorphism of human papillomavirus type 52 in women from Northeast China. Int J Mol Sci 13: 14962-14972, 2012.

22. Hang D, Yin Y, Han J, Jiang J, Ma H, Xie S, Feng X, Zhang K, $\mathrm{Hu} \mathrm{Z}$, Shen $\mathrm{H}$, et al: Analysis of human papillomavirus 16 variants and risk for cervical cancer in Chinese population. Virology 488: 156-161, 2016.

23. Shen M, Ding X, Li T, Chen G and Zhou X: Sequence variation analysis of HPV-18 Isolates in Southwest China. PLoS One 8: e56614, 2013

24. Prado JC, Calleja-Macias IE, Bernard HU, Kalantari M, Macay SA, Allan B, Williamson AL, Chung LP, Collins RJ, Zuna RE, et al: Worldwide genomic diversity of the human papillomaviruses-53,56, and 66, a group of high-risk HPVs unrelated to HPV-16 and HPV-18. Virology 340: 95-104, 2005.

25. Wyant PS, Cerqueira DM, Moraes DS, Leite JP, Martins CR, de Macedo Brígido M and Raiol T: Phylogeny and polymorphism in the long control region, E6, and L1 of human papillomavirus types 53, 56, and 66 in central Brazil. Int J Gynecol Cancer 21: 222-229, 2011.

26. Clarke KR and Gorley RN: Primer v5: User ManuallTutorial. PRIMER-E, Plymouth, p91, 2001.

27. Tamura K, Stecher G, Peterson D, Filipski A and Kumar S: MEGA6: Molecular evolutionary genetics analysis version 6.0. Mol Biol Evol 30: 2725-2729, 2013.

28. Buchan DW, Minneci F, Nugent TC, Bryson K and Jones DT: Scalable web services for the PSIPRED protein analysis workbench. Nucleic Acids Res 41: W349-W357, 2013.

29. Adzhubei IA, Schmidt S, Peshkin L, Ramensky VE, Gerasimova A, Bork P, Kondrashov AS and Sunyaev SR: A method and server for predicting damaging missense mutations. Nat Methods 7: 248-249, 2010 
30. Kel AE, Gössling E, Reuter I, Cheremushkin E, Kel-Margoulis OV and Wingender E: MATCHTM: A tool for searching transcription factor binding sites in DNA sequences. Nucleic Acids Res 31: 3576-3579, 2003.

31. Nei M and Gojoborit T: Simple methods for estimating the numbers of synonymous and nonsynonymous nucleotide substitutions. Mol Biol Evol 3: 418-426, 1986.

32. Burk RD, Harari A and Chen Z: Human papillomavirus genome variants. Virology 445: 232-243, 2013.

33. Schiller JT and Lowy DR: Understanding and learning from the success of prophylactic human papillomavirus vaccines. Nat Rev Microbiol 10: 681-692, 2012.

34. Pista A, Oliveira A, Barateiro A, Costa H, Verdasca N and Paixao MT: Molecular variants of human papillomavirus type 16 and 18 and risk for cervical neoplasia in Portugal. J Med Virol 79: 1889-1897, 2007.

35. Bernard HU: Regulatory elements in the viral genome Virology 445: 197-204, 2013

36. Moody CA and Laimins LA: Human papillomavirus oncoproteins: Pathways to transformation. Nat Rev Cancer 10: 550-560, 2010.

37. Thomas M, Narayan N, Pim D, Tomaić V, Massimi P, Nagasaka K, Kranjec C, Gammoh N and Banks L: Human papillomaviruses, cervical cancer and cell polarity. Oncogene 27: 7018-7030, 2008.

38. Chagas BS, Batista MV, Crovella S, Gurgel AP, Silva Neto Jda C, Serra IG, Amaral CM, Balbino VQ, Muniz MT and Freitas AC: Novel E6 and E7 oncogenes variants of human papillomavirus type 31 in Brazilian women with abnormal cervical cytology. Infect Genet Evol 16: 13-18, 2013.
39. Yao Y, Huang W, Yang X, Sun W, Liu X, Cun W and Ma Y: HPV-16 E6 and E7 protein T cell epitopes prediction analysis based on distributions of HLA-A loci across populations: An in silico approach. Vaccine 31: 2289-2294, 2013.

40. Chan PK, Zhang C, Park JS, Smith-McCune KK, Palefsky JM, Giovannelli L, Coutlée F, Hibbitts S, Konno R, Settheetham-Ishida W, et al: Geographical distribution and oncogenic risk association of human papillomavirus type 58 E6 and E7 sequence variations. Int J Cancer 132: 2528-2536, 2013.

41. Fleury MJ, Touze A and Coursaget P: Human papillomavirus type 16 pseudovirions with few point mutations in L1 major capsid protein FG loop could escape actual or future vaccination for potential use in gene therapy. Mol Biotechnol 56: 479-486, 2014.

42. Sapp M and Day PM: Structure, attachment and entry of polyoma- and papillomaviruses. Virology 384: 400-409, 2009.

43. Pande S, Jain N, Prusty BK, Bhambhani S, Gupta S, Sharma R, Batra $S$ and Das BC: Human papillomavirus type 16 variant analysis of E6, E7, and L1 genes and long control region in biopsy samples from cervical cancer patients in north India. J Clin Microbiol 46: 1060-1066, 2008.

This work is licensed under a Creative Commons Attribution 4.0 International (CC BY-NC 4.0) License 